\title{
Mechanistic and kinetic study of solar-light induced photocatalytic degradation of Acid Orange 7 in aqueous $\mathrm{TiO}_{2}$ suspensions
}

\author{
Maria Stylidi, Dimitris I. Kondarides, and Xenophon E. Verykios ${ }^{\dagger}$ \\ Department of Chemical Engineering, University of Patras, GR-26500 Patras, Greece
}

\begin{abstract}
The photocatalytic degradation of aqueous solutions of Acid Orange 7 in $\mathrm{TiO}_{2}$ suspensions has been investigated with the use of a solar light simulating source. It has been found that, when the full range of emitted photons is used, decolorization and complete mineralization of the solution is achieved with satisfactory rates, depending on initial dye concentration. A reaction pathway is proposed according to which degradation of the dye molecules adsorbed on the photocatalyst surface takes place via a series of oxidation steps, which lead to decolorization and formation of a number of intermediates, mainly aromatic and aliphatic acids, which are further oxidized toward compounds of progressively lower molecular weight. Eventually, mineralization is achieved leading to the formation of gas phase $\mathrm{CO}_{2}$ and inorganic ions in solution. Kinetic results show that the initial rate of decolorization depends strongly on surface coverage and on incident photon energy. Visible light photons contribute to decolorization, via the photosensitization mechanism, but with reaction rates which are more than two orders of magnitude lower than the corresponding ones induced by photons of energy higher than that of the band gap of the semiconductor. In both cases, the initial rate of decolorization is significantly reduced for dye coverages close to monolayer.
\end{abstract}

\section{INTRODUCTION}

Wastewaters generated by the textile industry are rated as the most polluting among all industrial sectors, considering both the volume rejected and the composition of the effluents [1]. Due to the large degree of chemical complexity, diversity and stability of the compounds contained in these wastewaters, conventional treatment methods are ineffective for their decolorization and degradation. This is particularly true for azo dyes, characterized by the presence of an azo group $(-\mathrm{N}=\mathrm{N}-)$, which constitute the largest and most important class of commercial dyes [1, 2]. Azo dyes, being mostly non-biodegradable under both natural and sewage treatment plant conditions [3], cause special environmental concern due to their toxicity and potential carcinogenic nature of their precursors and degradation products $[4,5]$.

Currently, extensive efforts are in progress to develop cleaner technologies and processes for the treatment of industrial effluents in order to meet the stringent international discharge standards and regulations (ISO 14001, October 1996). Among the various advanced oxidation processes (AOPs) which have been proposed for the treatment of recalcitrant compounds present in industrial wastewaters, including dyestuffs, heterogeneous photocatalysis is an attractive method, which has been successfully employed for the degradation of various families of organic pollutants [6-10]. The most important advantages of the photocatalytic method, compared to other AOPs, are related to the

${ }^{\dagger}$ E-mail: verykios@chemeng.upatras.gr possibility of carrying out the reaction under ambient conditions with the use of air as the oxidant, and to the use of largely available, inexpensive, non-toxic and stable photocatalysts (mainly $\mathrm{TiO}_{2}$ ). Most important, photocatalytic processes may utilize solar radiation, which is the environmentally ideal energy source.

The photocatalytic degradation of Acid Orange 7 (AO7) has been investigated by several groups [1119]. Vinodgopal et al. [11] studied the photosensitized degradation of $\mathrm{AO} 7$, and the kinetics of the visible light-induced reaction on the surface of $\mathrm{TiO}_{2}$ photocatalysts has been examined by Bauer et al. [12]. Lucarelli et al. [13] studied the photosensitized degradation of the azodye giving insight into the surface properties of different $\mathrm{TiO}_{2}$ samples used. Other research groups have studied the effect of operational parameters of the photocatalytic degradation of $\mathrm{AO} 7$ together with other azodyes using $\mathrm{TiO}_{2}$ [14-16] or other photocatalysts [17].

In our previous investigation [18], the effects of operational parameters on the photocatalytic degradation of aqueous solutions of $\mathrm{AO} 7$ were investigated, employing a solar light simulating source and $\mathrm{TiO}_{2}$-based catalysts. It was found that complete decolorization and substantial reduction of COD can be achieved with satisfactory rates with the use of optimal operational parameters. The photocatalytic reaction pathways of the $\mathrm{AO} 7-\mathrm{TiO}_{2}$-solar light system have been examined in detail recently [19], with the use of a variety of techniques, and $\mathrm{TiO}_{2}$-mediated photodegradation mechanism has been proposed on the basis of quantitative and qualitative detection of intermediate compounds. 
In the present study, the mechanistic steps involved in the photocatalytic degradation and mineralization of AO7 solutions are identified. Special emphasis is given on the effect of incident light energy (UV/vis versus visible) on the apparent decolorization rate of AO7 solutions, in order to quantify the extent to which the visible light-induced photosensitization mechanism [11, 20] contributes to the overall photocatalytic degradation of azo dyes by solar light radiation.

\section{EXPERIMENTAL}

2.1. Materials. The photocatalyst employed was titanium dioxide (ca. $80 \%$ anatase, $20 \%$ rutile; BET area, ca. $50 \mathrm{~m}^{2} \cdot \mathrm{g}^{-1}$; average particle size, $30 \mathrm{~nm}$ ) and was kindly supplied by Degussa Co (P25). Acid Orange 7 (Sigma Chemical Co.) was chosen as a model compound for the hydrosoluble dyes of the azo family and was used without further purification. When in solution, AO7 is present in two tautomeric forms (Scheme 1), the azo form and the hydrazone form, the latter being favored in aqueous solutions [21, 22]. Deionized, distilled water was used throughout this study.

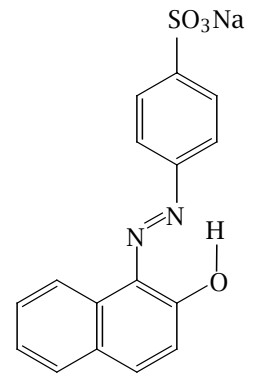

Azo form

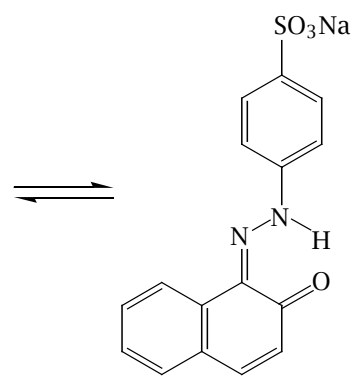

Hydrazone form
Scheme 1. Tautomeric forms of Acid Orange 7 in solutions.

2.2. Photoreactor and light sources. The apparatus employed in the photocatalytic experiments consists of the light source and the photoreactor, and has been described in detail elsewhere [18, 19]. The light source (Oriel, model 66021) is furnished with a solarlight simulating Xe-arc lamp (Osram XBO $450 \mathrm{~W}$ ), a set of lenses for light collection and focusing, and a water filter mounted on the lamp housing to eliminate infrared radiation. In certain experiments, a cutoff filter (Oriel, 51472) was placed in front of the water filter, to remove the UV portion of the radiation $(<400 \mathrm{~nm})$. The quartz photoreactor is equipped with optical flat light entry and exit windows and a top cover with provisions for in situ measurements of the $\mathrm{pH}$ and the conductivity of the solution [19]. Experiments were performed at room temperature, with a constant lamp power of $400 \mathrm{~W}$ and a fixed distance between the photoreactor and the lamp housing $(50 \mathrm{~cm})$. Under these conditions the photon flow entering the reactor, measured by chemical actinometry $[19,23]$, was found to be $9.5 \times 10^{-7}$ einsteins/sec and $7.6 \times 10^{-7}$ einsteins/sec in the absence and presence of the cutoff filter, respectively.

2.3. Procedures and analyses. In a typical experiment, a known amount of AO7 was dissolved in $70 \mathrm{ml}$ of distilled water contained in the photoreactor resulting in a solution with the desired dye concentration, which varied in the region of 5-600 mg AO7/L $\left(1.4 \times 10^{-5}-1.7 \times 10^{-3} \mathrm{M}\right)$. The $\mathrm{TiO}_{2}$ photocatalyst pow$\operatorname{der}(750 \mathrm{mg} / \mathrm{L})$ was then added to the solution under continuous stirring and the suspension was left in the dark for 30 minutes to ensure establishment of adsorption/desorption equilibrium. At $t=0$ light was allowed to irradiate the reactor and samples were taken at given time intervals, centrifuged in a Heraeus Sepatech Labofuge at $4000 \mathrm{rpm}$ for $10 \mathrm{~min}$ and subsequently filtered through a Millipore filter (pore size $0.45 \mu \mathrm{m}$ ) to remove $\mathrm{TiO}_{2}$ particles [19]. The filtrates were analyzed by UV/vis spectroscopy with a Hitachi (model U 2001) spectrophotometer. Chemical oxygen demand (COD) measurements were obtained by the closed reflux titrimetric method [24].

Identification of reaction intermediates was accomplished with the use of GC/MS. Spectra were obtained with a gas chromatograph (Hewlett Packard 6890), equipped with an HP-1MS capillary column $(30 \mathrm{~m} \times$ $0.25 \mathrm{~mm} \times 1.0 \mu \mathrm{m}$ film thickness), interfaced directly to the mass spectrometer (HP 5973) used as a detector. Preparation of samples and methods employed for GC/MS measurements has been described in detail elsewhere [19]. Ion chromatography (Dionex Corp. Sunnyvale, Calif.) was used to determine the quantity of $\mathrm{NO}_{3}{ }^{-}$ and $\mathrm{SO}_{4}{ }^{2-}$ ions as well as the low molecular weight aliphatic acids. The concentration of $\mathrm{NH}_{4}{ }^{+}$ions in solution was measured by the phenate method, and the rate of evolution of $\mathrm{CO}_{2}$ was monitored with the use of a NDIR $\mathrm{CO}_{2}$ analyzer (Rosemount Binos 100). Details on the above methods can be found elsewhere [19].

2.4. Equilibrium adsorption measurements. The adsorption isotherm of $\mathrm{AO} 7$ on $\mathrm{TiO}_{2}$ was obtained at room temperature and natural $\mathrm{pH}$ by determining the amount of the dye adsorbed on the photocatalyst surface as a function of its concentration in the solution, at equilibrium. For this, a fixed weight of $\mathrm{TiO}_{2}(200 \mathrm{mg})$ was added in a series of $100 \mathrm{~mL}$ aliquots of aqueous solutions of $\mathrm{AO} 7$ of variable concentration, in the range of $1 \times 10^{-4}-6 \times 10^{-3} \mathrm{M}$. After sonication for $15 \mathrm{~min}$ utes, the suspensions were left overnight (12 hours) in the dark under continuous stirring. The samples were filtered using a $0.45 \mu \mathrm{m}$ filter and the concentration of the dye in the filtrate was determined by measuring the absorbance at $485 \mathrm{~nm}$. 
The amount of the dye molecules adsorbed on $\mathrm{TiO}_{2}, N_{\text {ads }}(\mathrm{mol} / \mathrm{g})$, was calculated from the following equation:

$$
N_{\mathrm{ads}}=\frac{V \Delta C}{w},
$$

where $\Delta C=C_{0}-C_{\text {eq }}(\mathrm{mol} / \mathrm{L})$ is the decrease in the molarity of AO7 after reaching equilibrium, with $C_{0}$ being the initial concentration, $C_{\text {eq }}$ the AO7 concentration at equilibrium, $V(\mathrm{~L})$ the volume of aqueous solution and $w(\mathrm{~g})$ the mass of the oxide. The concentration of the dye in the supernatant was determined spectrophotometrically and subtracted from the initial concentration to determine the adsorbed amount in each case.

\section{RESULTS AND DISCUSSSION}

3.1. Adsorption isotherm of AO7 on $\mathrm{TiO}_{2}$. The adsorption isotherm of $\mathrm{AO} 7$ on $\mathrm{TiO}_{2}$ is presented in Figure 1(A), in which the amount of dye adsorbed on the photocatalyst surface is plotted as a function of its concentration in the solution, at equilibrium. It is observed that $N_{\text {ads }}$ increases with increasing $C_{\text {eq }}$, and reaches a plateau at equilibrium concentrations above $1.5 \times 10^{-3} \mathrm{~mol} / \mathrm{L}$. It may be assumed that this plateau corresponds to monolayer coverage of the dye on the titanium oxide surface. Increasing equilibrium concentrations above $2.0 \times 10^{-3} \mathrm{~mol} / \mathrm{L}$ results in the adsorption of additional amounts of AO7, which may be attributed to the formation of additional layers.

Adsorption of $\mathrm{AO} 7$ on $\mathrm{TiO}_{2}$ at monolayer coverage can be expressed by the following equation:

$$
N_{\mathrm{ads}}=\frac{N_{\mathrm{max}} K C_{\mathrm{eq}}}{1+K C_{\mathrm{eq}}},
$$

where $N_{\max }$ is the total number of accessible adsorption sites, per gram of $\mathrm{TiO}_{2}$, and $K$ represents the equilibrium adsorption constant for the process of dye adsorption. The linearized form of eq. (2) is the following:

$$
\frac{C_{\mathrm{eq}}}{N_{\mathrm{ads}}}=\frac{1}{K N_{\max }}+\frac{C_{\mathrm{eq}}}{N_{\max }} .
$$

As observed in Figure 1(B), the plot of $C_{\text {eq }} / N_{\text {ads }}$ versus $C_{\text {eq }}$ results in a straight line, from the intercept and slope of which, the values of $N_{\max }=4.66 \times$ $10^{-5} \mathrm{~mol} \cdot \mathrm{g}^{-1}$ and $K=10360 \mathrm{~L} \cdot \mathrm{mol}^{-1}$ are calculated. Assuming that a complete monolayer is formed at total adsorption, $N_{\max }$ is equal to:

$$
N_{\text {max }}=\frac{A_{\mathrm{sp}}}{N_{A} \sigma^{0}},
$$

where $A_{\mathrm{sp}}$ is the adsorbent specific surface area, $N_{A}$ is Avogadro's number and $\sigma^{0}$ is the area that each dye molecule occupies on the surface at monolayer
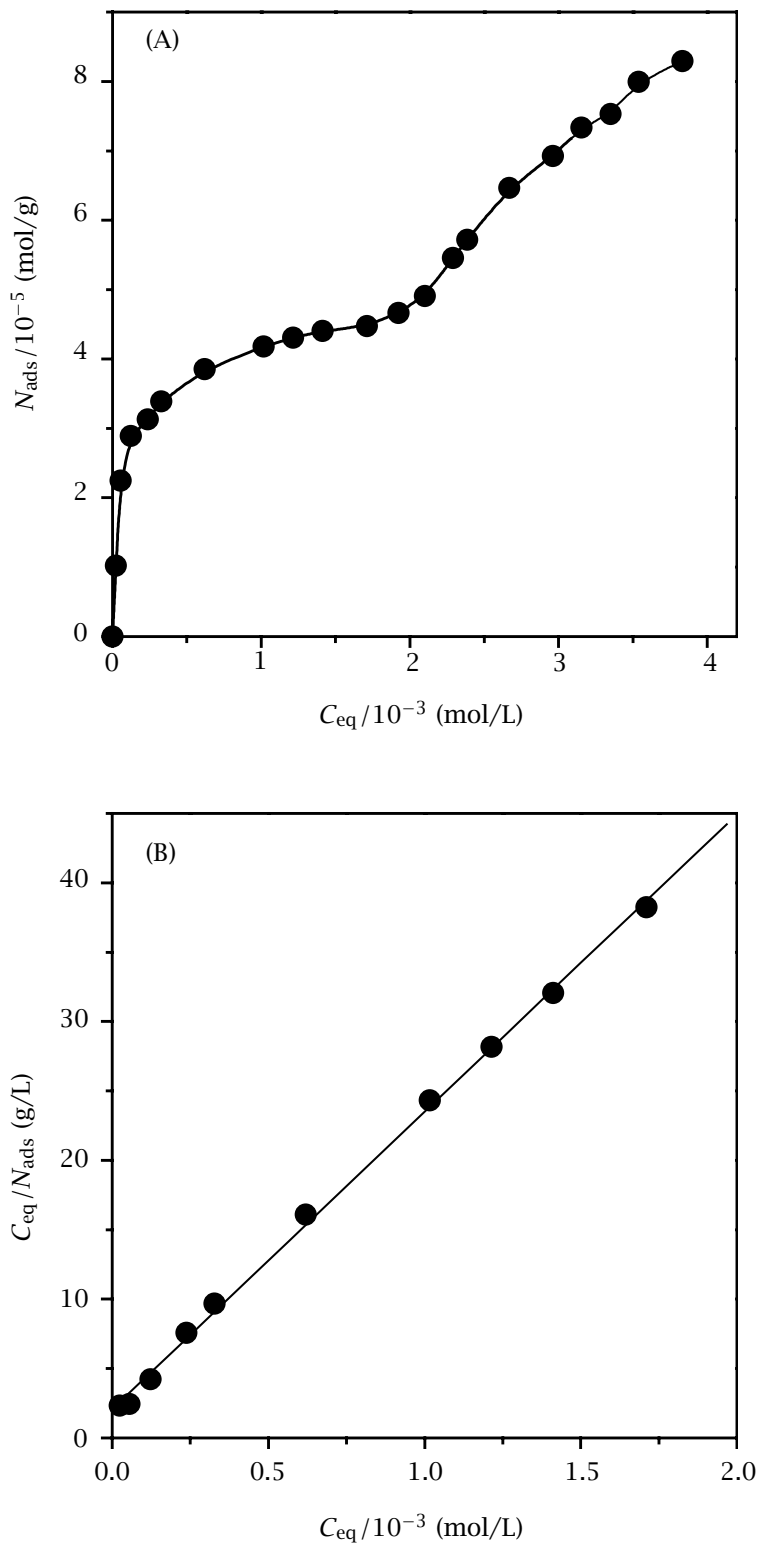

Figure 1. (A) Adsorption isotherm for AO7 on $\mathrm{TiO}_{2}$ (P25) at room temperature. (B) Same data plotted in the linearized form, according to eq. (3).

coverage. Given that $N_{\max }=4.66 \times 10^{-5} \mathrm{~mol} \cdot \mathrm{g}^{-1}$ and $A_{\mathrm{sp}}=50 \mathrm{~m}^{2} \cdot \mathrm{g}^{-1}$, a value of $\sigma^{0}=178 \AA^{2}$ is calculated. Based on the bond lengths and angles between the atoms that constitute the dye molecule, the projectional surface area of AO7 molecule is calculated to be $62.2 \AA^{2}$ [25], which is about one third of the experimental value calculated above. This means that, under the experimental conditions employed, only a portion of the surface area of $\mathrm{TiO}_{2}(\sim 36 \%)$ is accessible to AO7 adsorption, which can be attributed to stereochemical restrictions and/or to the presence of coadsorbed water molecules on the photocatalyst surface. 


\subsection{Mechanism of photocatalytic degradation of} AO7 by solar light. The mechanism of photocatalytic degradation of AO7 by solar light was investigated using a dye concentration of $300 \mathrm{mg} / \mathrm{L}\left(C_{0}=\right.$ $\left.8.5 \times 10^{-4} \mathrm{M}\right)$, at natural $\mathrm{pH}(5.8)$. The concentration of the solution was deliberately high in order to allow for qualitative and quantitative determination of reaction intermediates. The relative concentration of the dye, determined by the absorbance of the hydrazone form of the dye at $485 \mathrm{~nm}$, and the COD of the solution as functions of time of irradiation are shown in Figure 2. It is observed that the dye concentration decreases exponentially with time and diminishes after approximately 10 hours, resulting in complete decolorization of the solution. The chemical oxygen demand decreases rather slowly during the first 10 hours under irradiation, i.e., when the solution is still colored. This indicates that decolorization is due to decomposition of AO7 molecules to lower molecular weight compounds, which still contribute to the COD of the solution. After complete decolorization is achieved, the COD decreases rapidly and drops to less than $10 \%$ of its initial value after 15 hours (Figure 2). Prolonged irradiation of the suspension results in elimination of the COD of the solution, indicating that complete mineralization occurs after 22-25 hours.

The investigation of the formation and fate of photodegradation products of AO7 was accomplished with the use of GC/MS spectroscopy and ion chromatography and revealed the existence of more than twenty different reaction intermediates [19]. The evolution of some typical compounds in solution is presented in

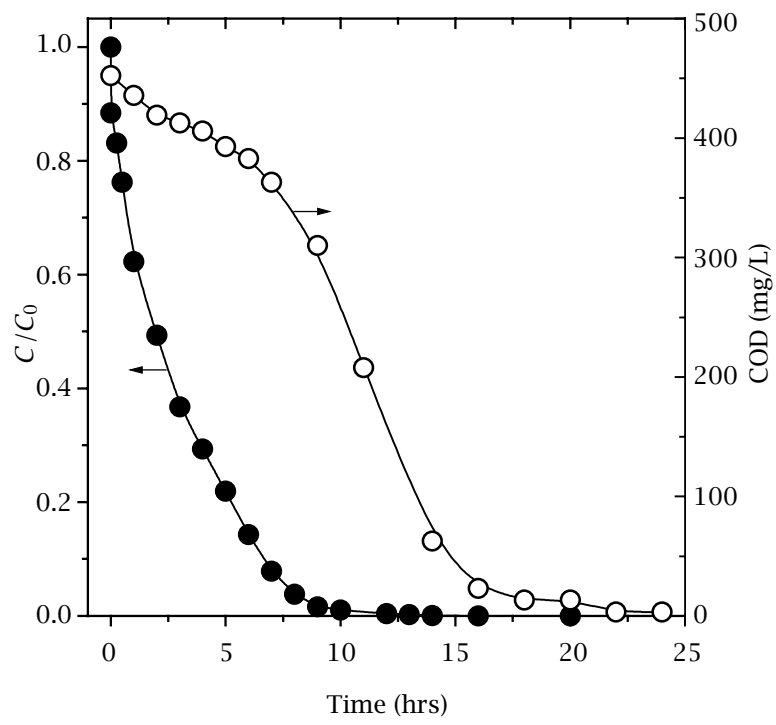

Figure 2. Decolorization and decrease of COD of an aqueous solution of $A O 7\left(C_{0}=300 \mathrm{mg} / \mathrm{L}\right)$ as functions of time of irradiation with solar light.
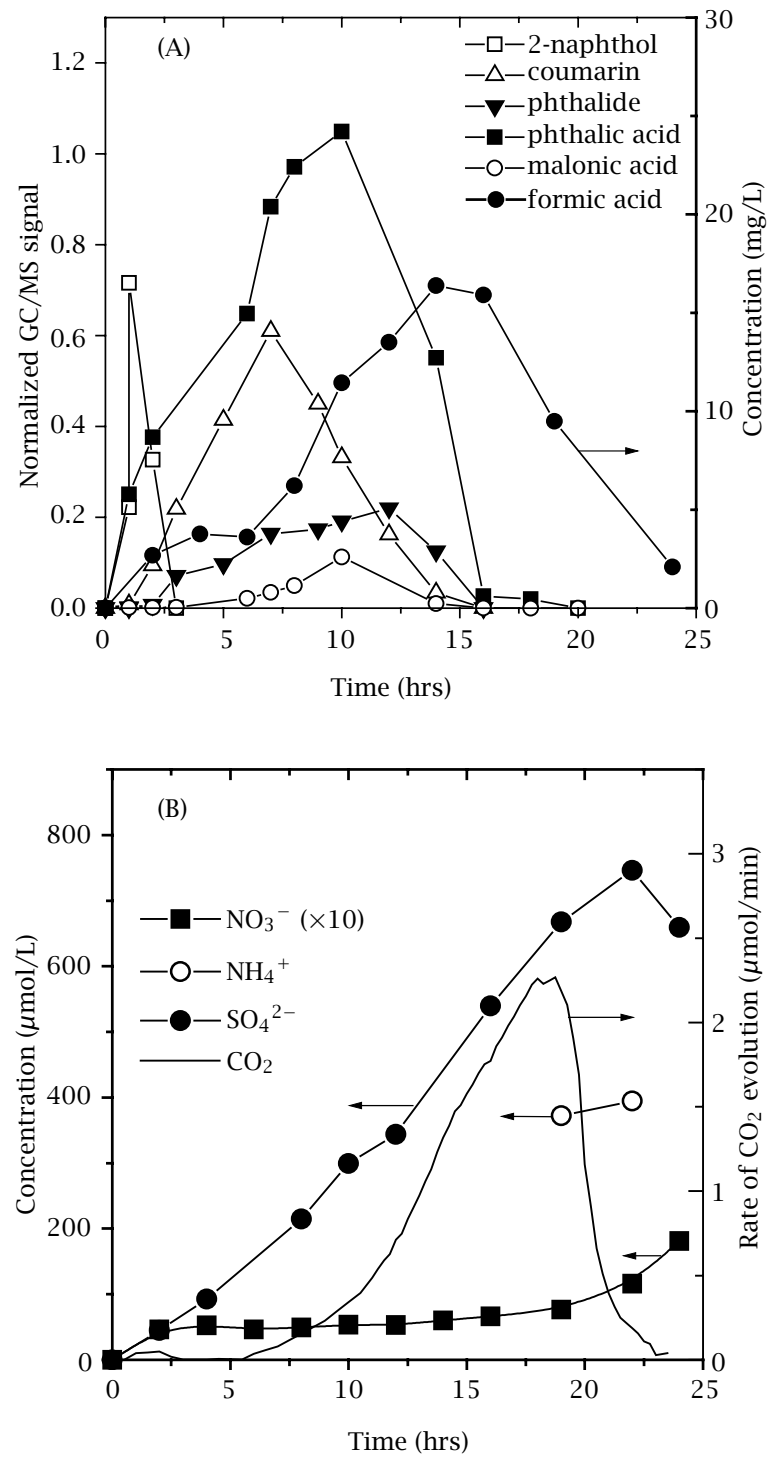

Figure 3. Evolution of (A) selected reaction intermediates and (B) mineralization products as functions of time of irradiation with solar light.

Figure 3(A), where the normalized areas of the corresponding GC/MS signals are plotted as functions of irradiation time. Normalization has been done with respect to the GC/MS peak area of cyclohexane, which has been added to the sample under freezing conditions and used as an internal standard [19]. Cyclohexane was chosen because, under the experimental conditions employed, it gives a very well separated, sharp peak which does not interfere with chromatographic peaks of the compounds contained in the samples under investigation. Quantitative determination of low molecular weigh acids, such as formic acid (Figure 3(A)), was accomplished by ion chromatography. The evolution of mineralization products is shown in Figure 3(B), where the concentrations of $\mathrm{NO}_{3}{ }^{-}, \mathrm{NH}_{4}{ }^{+}$and $\mathrm{SO}_{4}{ }^{2-}$ ions, as 

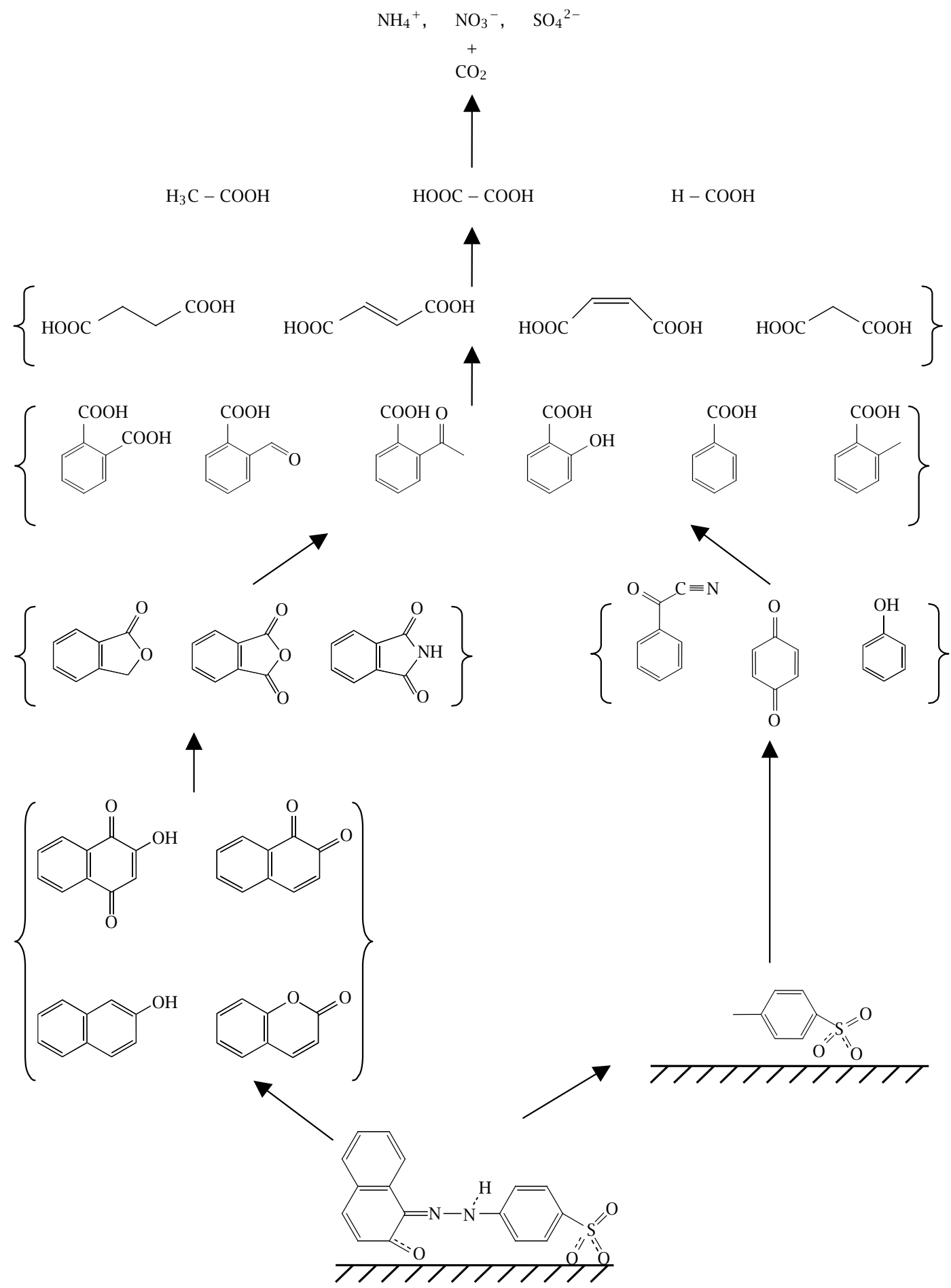

Figure 4. Pathways of photocatalytic degradation of AO7 by solar light.

well as the rate of evolution of $\mathrm{CO}_{2}$ in the gas phase are plotted as functions of time of irradiation.

Based on the present and previous results [19], the reaction pathways for photocatalytic degradation of aqueous solutions of $\mathrm{AO} 7$ by solar radiation have been identified, as schematically shown in Figure 4. It should be noted that the presence of all intermediates and final products shown in Figure 4 has been verified with the use of appropriate techniques [19]. According to the proposed reaction scheme, AO7 adsorbs on the 
photocatalyst surface to an extent which depends on initial dye concentration in solution (Figure 1). Based on ex situ FTIR measurements of the present system [19], and in agreement with the results of Bauer et al. [26], it is proposed that adsorption occurs via the oxygen of the hydrazone form $(\mathrm{C}=\mathrm{O})$ and the two oxygen atoms of the sulfonate group of the dye molecule (Figure 4).

Irradiation of the $\mathrm{AO} 7 / \mathrm{TiO}_{2}$ with the solar light simulating source results, initially, in the formation of naphthalene-type molecules, such as 2-naphthol and coumarin (Figure 3(A)), which may be considered as primary intermediate products. These compounds originate from oxidative cleavage of the dye molecule in the vicinity of the azo bond (Figure 4), a process which is responsible for the decolorization of the solution (Figure 2). Smaller aromatic intermediates containing a sixand a five-atom ring, such as phthalide, appear in the continue and are further oxidized to yield smaller fragments, such as phthalic acid (Figure 3(A)). Eventually, aliphatic acids, including malonic (Figure 3(A)), fumaric, succinic and maleic acid appear in the solution, with their concentration going through maxima at ca. 10 hours under irradiation. At this point, the solution is decolorized and the COD starts to decrease with sufficiently higher rates (Figure 2). This is accompanied by the formation of low molecular weight acids, such as formic acid (Figure 3(A)), which eventually decompose to yield carbon dioxide (Figure 3(B)). Formation of $\mathrm{CO}_{2}$ probably takes place by decarbonylation of carboxylic acids via the "photo-Kolbe" reaction [27]:

$$
\mathrm{R}-\mathrm{COO}^{-}+\mathrm{h}^{+} \longrightarrow \mathrm{R}^{0}+\mathrm{CO}_{2}
$$

The total amount of $\mathrm{CO}_{2}$ that evolves in the gas phase (960 $\mu \mathrm{mol})$ is in excellent agreement with the theoretical amount of $952 \mu \mathrm{mol}$ that should be formed following deep oxidation of all AO7 molecules contained in the initial solution. This is also reflected in the decrease of COD to zero values at the end of the experiment (Figure 2). The fact that the release of $\mathrm{CO}_{2}$ in the gas phase is delayed (Figure 3(B)), compared to the decrease of $\mathrm{COD}$, may be attributed to the slow desorption of $\mathrm{CO}_{2}$ from the catalyst surface to the solution and its subsequent diffusion to the gas phase.

It should be noted that no sulfur-containing compounds, such as benzene sulfonate, were observed in solution, which gives evidence that the primary intermediate which results from the initial cleavage of the dye is strongly held on the photocatalyst surface via the oxygen atoms of the sulfonate group (Figure 4). However, this fragment is subjected to degradation and mineralization, as evidenced by the evolution of $\mathrm{SO}_{4}{ }^{2-}$ ions in solution. The concentration of the latter is ca. $20 \%$ lower than the expected from stoichiometry, indicating that, possibly, an amount of sulfur ions remain adsorbed on the photocatalyst surface. Regarding the concentration of the nitrogen-containing ions
$\left(\mathrm{NH}_{4}{ }^{+}, \mathrm{NO}_{3}{ }^{-}\right)$in solution at the end of the experiment, it is about four times less than the expected from stoichiometry, indicating that either N-containing species remain adsorbed on the photocatalyst surface or, most probably, that significant amounts of $\mathrm{N}_{2}$ and/or $\mathrm{NH}_{3}$ have been produced and transferred to the gas phase.

3.3. Kinetics of decolorization of AO7 solutions. The kinetics of decolorization of aqueous solutions of AO7 was examined using different initial dye concentrations. Results obtained following irradiation of the $\mathrm{AO} 7-\mathrm{TiO}_{2}$ suspensions with the solar light simulating source are shown in Figure 5(A), where the normalized concentrations of AO7 are plotted as functions of initial
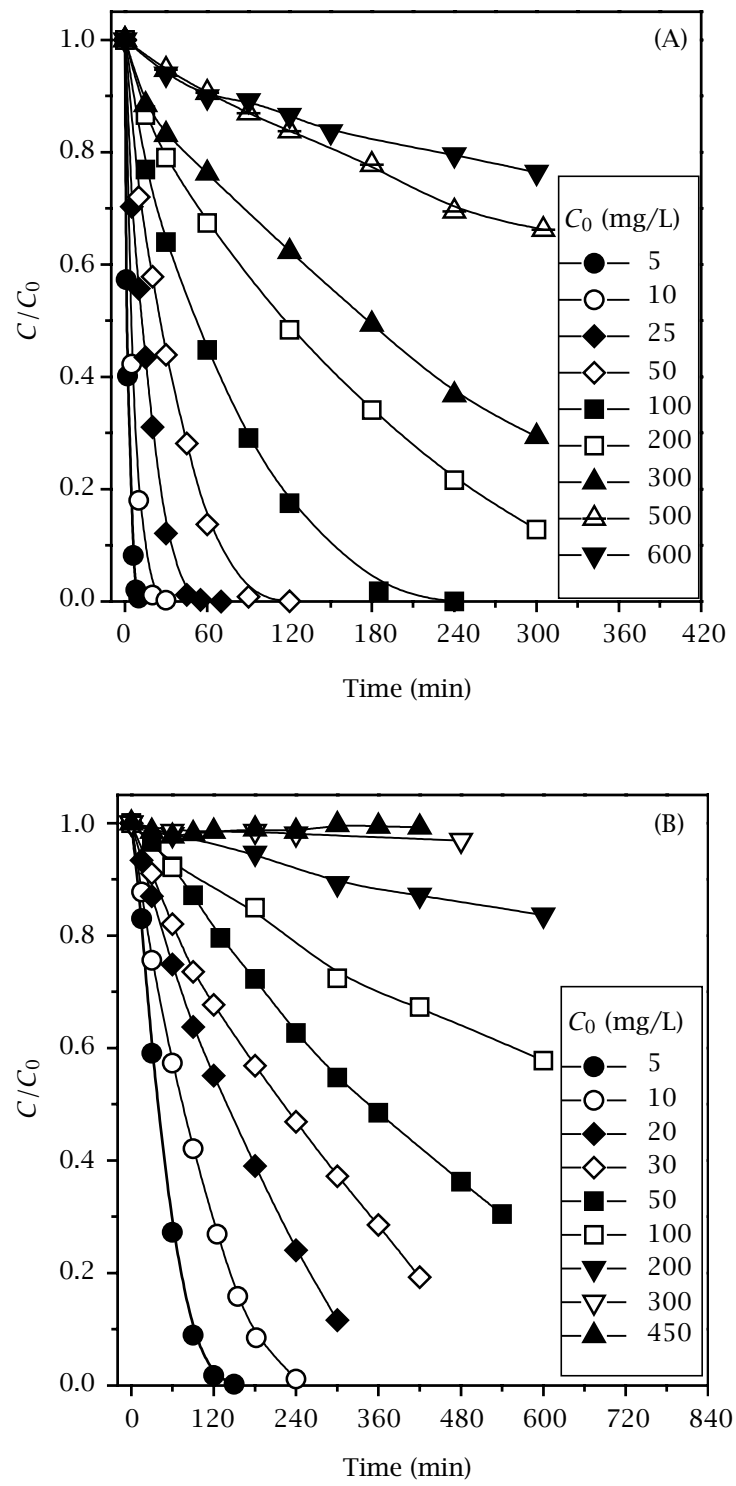

Figure 5. Effect of initial dye concentration on the decolorization of aqueous solutions of AO7 irradiated with (A) solar and (B) visible (> $400 \mathrm{~nm}$ ) light. 
dye concentration. Normalization was done with respect to the dye concentration in solution obtained after 30 minutes in the dark (equilibrium concentration). It should be noted that, under all experimental conditions employed, decolorization of the solution does not take place in the absence of photocatalyst or in the dark. It is observed that the time required for the decolorization of the AO7 solutions depends strongly on the initial dye concentration. Complete decolorization occurs in less than three hours for relatively low $C_{0}$ values (5-100 $\mathrm{mg} / \mathrm{L})$ while significantly longer time periods are required for higher dye concentrations (200-600 mg/L).

Similar results obtained with the use of the cutoff filter, which permits transmittance of photons with wavelengths higher than $400 \mathrm{~nm}$, are presented in Figure 5(B). It is observed that decolorization curves are qualitatively similar to those obtained with the use of $\mathrm{UV} /$ vis radiation (Figure 5(A)) but the time required for decolorization is significantly longer in the present case. However, complete decolorization is achieved within a few hours of irradiation for low initial dye concentrations $(5-10 \mathrm{mg} / \mathrm{L})$, while substantial color degradation is observed for $C_{0}$ values up to $50-100 \mathrm{mg} / \mathrm{L}$. Results of Figure 5(B) can be explained considering that, although $\mathrm{TiO}_{2}$ cannot be directly activated by photons of $\lambda>400 \mathrm{~nm}$, visible light can induce the excitation of the dye molecule. The excited dye injects an electron to the conduction band of $\mathrm{TiO}_{2}$ particles because the oxidation potential of excited AO7 (for its singlet state) is more negative than the potential of the conduction band of $\mathrm{TiO}_{2}$ particles under neutral conditions and, therefore, the electron transfer is thermodynamically allowed [28, 29].

The first points of the dye degradation curves of Figure 5 were used to determine the initial (apparent) decolorization rate of $\mathrm{AO} 7$ as a function of initial dye concentration, for both UV/vis- and visible-light illumination conditions, and results are plotted in Figure 6(A). It should be mentioned again that owing to adsorption of $\mathrm{AO} 7$ on the $\mathrm{TiO}_{2}$ surface, there is a slight difference in concentration before and after addition of the photocatalyst to the dye solution. Consequently, $C_{\text {eq }}$, rather than the initial concentration $C_{0}$, represents the true equilibrium concentration of AO7 in solution and, therefore, used in the present graph. It is observed that, in general, the decolorization rate is about 20 times higher for the UV/vis-irradiated system, compared to the visible-irradiated one. Considering that the intensity of the incident light is reduced by only $20 \%$ when the cutoff filter is used (see experimental section), the observed differences should be mainly attributed to the effect of the incident photon energy on the decolorization rate or, equivalently, to the different efficiency of the charge separation mechanism versus the photosensitization mechanism. Taking into account that the portion of UV radiation $(\lambda<400 \mathrm{~nm})$ emitted from the lamp employed is much less than $20 \%$ of the total, it is concluded that the decolorization rate induced by these high energy photons is more than two orders of magnitude higher than that of photons of $\lambda>400 \mathrm{~nm}$.

An alternative way to present the data of Figure $6(\mathrm{~A})$ is to plot the reaction rates as functions of initial surface coverage, using the adsorption isotherm results of Figure 1, as shown in Figure 6(B). It is observed that when solar light is used (UV/vis), the initial rate of decolorization decreases by ca. $40 \%$ with increasing coverage from 0.1 to 0.4 and remains stable upon further increasing coverage to 0.9. However, as the coverage increases close to monolayer the reaction rate is drastically reduced. When only visible light is used $(\lambda>400 \mathrm{~nm})$, the initial rate of decolorization is almost constant for surface coverages in the region of
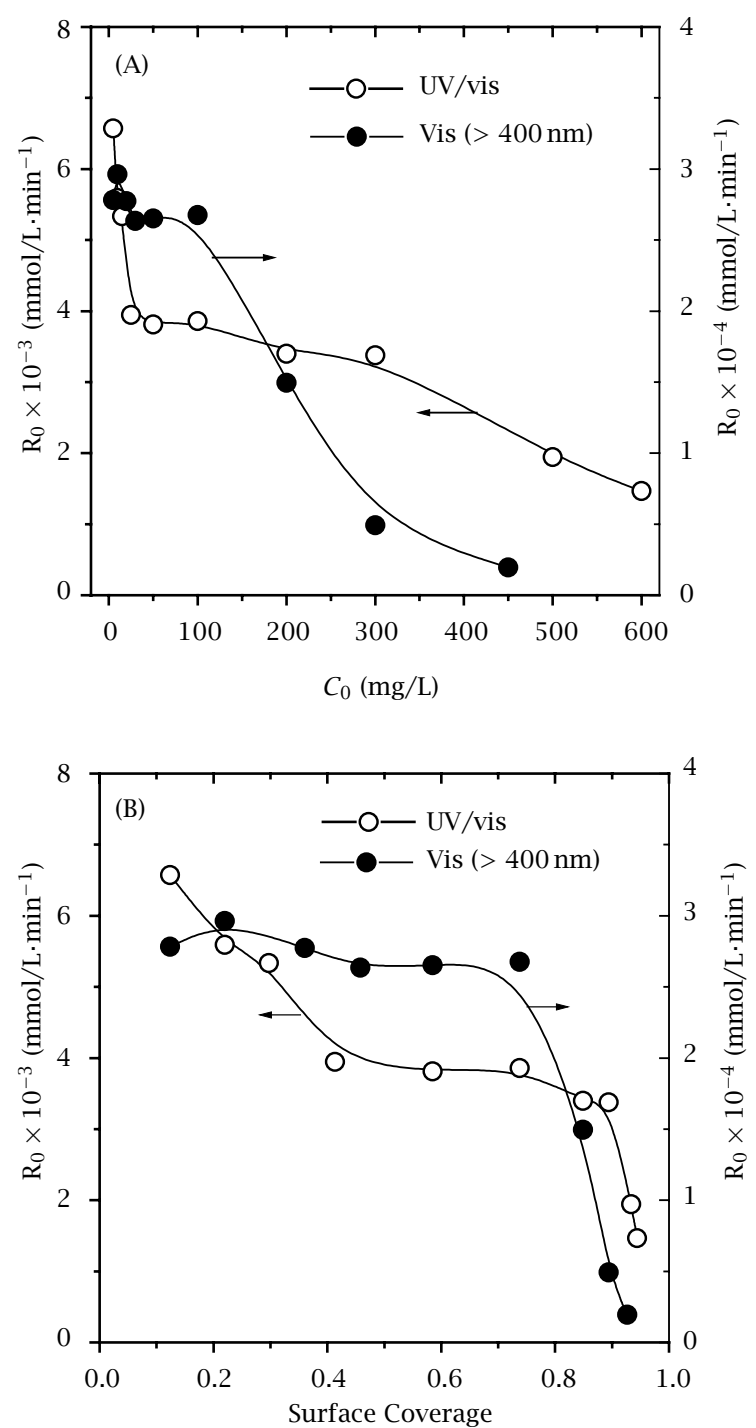

Figure 6. (A) Effect of dye concentration on the initial rate of decolorization of aqueous solutions of AO7 irradiated with solar or visible (> $400 \mathrm{~nm}$ ) light. (B) Same reaction rates plotted as functions of surface coverage. 
$0.1<\theta<0.7$ (Figure 6(B)). For $\theta>0.7$ the rate decreases sharply with increasing coverage and drops to about $5 \%$ of its initial value close to monolayer coverages.

The different dependence of the apparent decolorization rates observed for the UV/vis- and visiblelight irradiated systems on surface coverage can be explained considering that two different mechanisms are operable, depending on the energy of the incident photons. However, it should be kept in mind that the measured rates (Figure 6), determined by the changes of dye concentration in solution, are apparent, overall rates, including adsorption of the dye molecules on the photocatalyst, interaction of photons with the semiconductor (or adsorbed dye molecules), surface reactions, desorption of intermediate species, etc.

In the case of the UV/vis irradiated system, the decolorization rate is, in general, proportional to the rate of generation of valence band holes and conduction band electrons, which, in turn, depends on the probability of an incident photon to interact with a "free" site of the semiconductor surface. The observed decrease of decolorization rate with increasing surface coverage (Figure 6(B)) may then be attributed to the lower probability of photoinduced charge separation caused by the presence of adsorbed dye molecules. When the surface coverage reaches that of a monolayer, a large fraction of the incident light is captured by the adsorbed molecules and never reaches the photocatalyst surface to induce charge separation of the semiconductor, resulting in the observed abrupt decrease of the decolorization rate.

When only visible light is used, one might expect the rate of decolorization to be proportional to the amount of adsorbed dye molecules, which is not true in the present case (Figure 6(B)). The fact that the apparent reaction rate does not depend on coverage in the region of $0.1<\theta<0.7$ indicates that a compensation effect is operable, which acts in the opposite direction. This could be due to the fact that, as discussed above, visible-light photons are not able to further oxidize the fragments of the dye molecules produced, which accumulate on the photocatalyst surface and, therefore, hinder further adsorption of dye molecules from the solution. This reasoning may also explain the drastic decrease of decolorization rate at dye coverages which are significantly lower than in the case of the UV/vis system (Figure 6(B)). Accumulation of such fragments and/or formation of multilayers inhibits the reaction of dye molecules since there is not a direct contact of the semiconductor with them. The effect of surface coverage on the visible light-induced decolorization rate of AO7 is currently under detailed investigation.

3.4. Contribution of visible light radiation to the mineralization of AO7 solutions. Results of Figure 5(B) and 6 show that decolorization of aqueous solutions of AO7 can be achieved with relatively slow but appreciable rates, at least for low dye concentrations. However, from a practical point of view, not only decolorization but also COD removal is of interest. In Figure 7 are shown the changes of relative concentration of the dye and of the COD of a relatively low-initial-concentration solution $\left(C_{0}=50 \mathrm{mg} / \mathrm{L}\right)$ as functions of time of irradiation. It is observed that the dye concentration decreases with time and diminishes after about 20 hours under illumination, resulting in complete decolorization of the solution. The rate of COD removal is much lower than that of decolorization and, in contrast to what observed when $\mathrm{UV} /$ vis radiation was used (Figure 2), it does not increase when complete decolorization of the solution is achieved. This is expected since the photosensitization mechanism is operable only when dye molecules are present on the photocatalyst surface. When this is the case, electron injection from the excited molecule into the conduction band of the semiconductor takes place, and the dye molecule converts into its cationic radical $[13,14]$. The injected electron may then reduce surface chemisorbed oxidants, usually oxygen, to yield oxidizing species $\left(\mathrm{O}_{2}{ }^{\bullet-}\right.$, $\mathrm{HOO}^{\bullet}$ and $\mathrm{OH}^{\bullet}$ radicals) which can cause photodegradation. The action of the latter species causes, eventually, the small decrease of COD observed during the first 20 hours under irradiation (Figure 7). However, once the visible light chromophore is destroyed, the system can no longer absorb visible light, the oxidizing species are not formed and the COD of the solution does not further decrease (Figure 7).

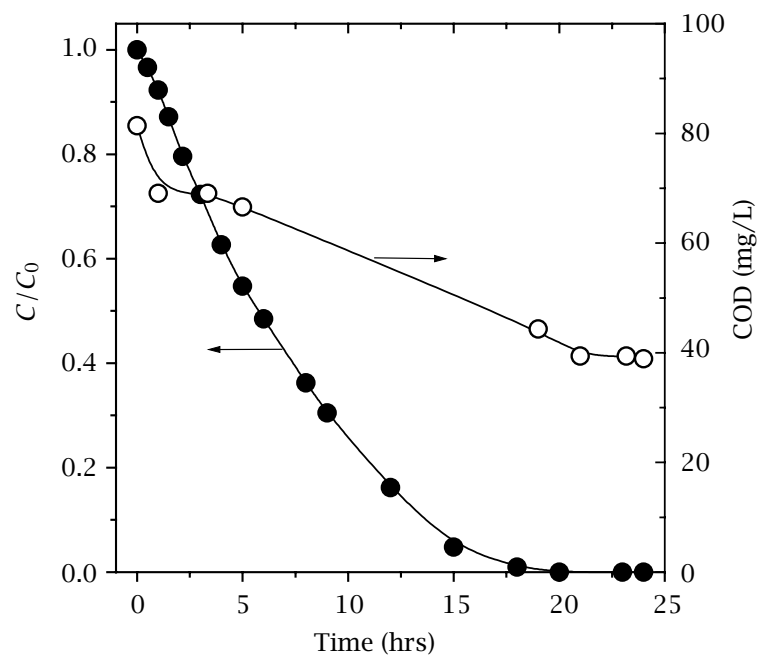

Figure 7. Decolorization and decrease of COD of an aqueous solution of $A O 7\left(C_{0}=50 \mathrm{mg} / \mathrm{L}\right)$ as functions of time of irradiation with visible light $(\lambda>400 \mathrm{~nm})$.

\section{CONCLUSIONS}

Irradiation of aqueous solutions of AO7 with artificial solar light radiation, in the presence of $\mathrm{TiO}_{2}$ photocatalyst, results in decolorization and, eventually, to 
complete mineralization of the solution. A reaction pathway is proposed according to which degradation of the dye molecules is achieved via a series of successive oxidation steps, which lead to the formation of aromatic and aliphatic acids of progressively lower molecular weight and, finally, to mineralization products (gas phase $\mathrm{CO}_{2}$ and $\mathrm{SO}_{4}{ }^{2-}, \mathrm{NH}_{4}{ }^{+}$and $\mathrm{NO}_{3}{ }^{-}$ions in solution). Kinetic results show that the initial rate of decolorization depends strongly on surface coverage. It decreases by about $40 \%$ with increasing surface coverage from 0.1 to 0.4 , remains stable for $0.4<\theta<0.9$, and is significantly reduced for coverages close to monolayer.

Visible light photons contribute to decolorization, via the dye photosensitization mechanism, but with reaction rates which are, typically, more than two orders of magnitude lower than the corresponding ones measured in the presence of photons with energy higher than that of the band gap of the semiconductor. When only visible light is used, initial decolorization rates are almost independent of surface coverage for $0.1<\theta<$ 0.7 , and are drastically reduced for higher coverages. The contribution of photons with $\lambda>400 \mathrm{~nm}$ to the decrease of COD of the solution is relatively small when the solution is still colored and diminishes when the visible light chromophore is destroyed.

\section{REFERENCES}

[1] R. Reid, J. Soc. Dyers Color. 112 (1996), 103.

[2] H. Zollinger, Color Chemistry: Synthesis, Properties and Applications of Organic Dyes and Pigments, VCH Publishers, New York, 1991.

[3] U. Pagga and D. Brown, Chemosphere 15 (1986), 476.

[4] K.-T. Chung and C. E. Cerniglia, Mutat. Res. 277 (1992), 201.

[5] R. Nillson, R. Nordlinder, and U. Wass, British J. Ind. Medi. 50 (1993), 65.

[6] O. Legrini, E. Oliveros, and A. M. Braun, Chem. Rev. 93 (1993), 671.

[7] M. A. Fox and M. Dulay, Chem. Rev. 93 (1993), 341.

[8] M. R. Hoffmann, S. T. Martin, W. Choi, and D. W. Bahnemann, Chem. Rev. 95 (1995), 69.

[9] D. F. Ollis and H. Al-Ekabi (Eds.), Photocatalytic Purification and Treatment of Water and Air, Elsevier Science Publishers, Amsterdam, 1993.
[10] C. S. Turchi and D. F. Ollis, J. Catal. 122 (1990), 178.

[11] K. Vinodgopal, D. E. Wynkoop, and P. V. Kamat, Environ. Sci. Technol. 30 (1996), 1660.

[12] C. Bauer, P. Jacques, and A. Kalt, J. Photochem. Photobiol. A: Chem. 140 (2001), 87.

[13] L. Lucarelli, V. Nadtochenko, and J. Kiwi, Langmuir 16 (2000), 1102.

[14] M. S. T. Gonçalves, A. M. F. Oliveira-Campos, E. M. M. S. Pinto, P. M. S. Plasência, and M. J. R. P. Queiroz, Chemosphere 39 (1999), 781.

[15] K. Tanaka, K. Padermpole, and T. Hisanaga, Wat. Res. 34 (2000), 327.

[16] C. Hachem, F. Bocquillon, O. Zahraa, and M. Bouchy, Dyes and Pigments 49 (2001), 117.

[17] F. Chen, Y. Xie, J. Zhao, and G. Lu, Chemosphere 44 (2001), 1159.

[18] F. Kiriakidou, D. I. Kondarides, and X. E. Verykios, Cat. Today 54 (1999), 119.

[19] M. Stylidi, D. I. Kondarides, and X. E. Verykios, Appl. Catal. B (2002), in press.

[20] M. S. Dieckmann and K. A. Gray, Wat. Res. 30 (1996), 1169.

[21] J. Griffiths and C. Hawkins, J. Chem. Soc. Perkin Trans. 2 (1977), 747.

[22] N. Kawashima, N. Fujimoto, and K. Meguro, J. Colloid Interface Sci. 103 (1985), 459.

[23] S. L. Murov, I. Carmichael, and G. L. Hug (Eds.), Handbook of Photochemistry, second edition, Dekker, New York, 1993.

[24] A. D. Eaton, L. S. Clesceri, and A. E. Greenberg (Eds.), Standard Methods for the Examination of Water and Wastewater, $19^{\text {th }}$ edition, American Public Health Association, Washington DC, 1995.

[25] A. B. Tsevis, Ph.D. Thesis, Dept. of Chem. Eng., University of Patras, Greece, 2002.

[26] C. Bauer, P. Jacques, and A. Kalt, Chem. Phys. Lett. 307 (1999), 397.

[27] M. Vautier, C. Guillard, and J.-M. Herrmann, J. Catal. 201 (2001), 46.

[28] J. Bandara, J. A. Mielczarski, and J. Kiwi, Langmuir 15 (1999), 7680.

[29] F. Zhang, J. Zhao, T. Shen, H. Hidaka, E. Pelizzetti, and N. Serpone, Appl. Catal. B 15 (1998), 147. 


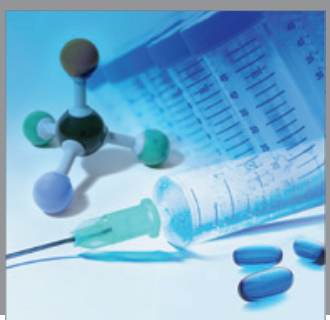

International Journal of

Medicinal Chemistry

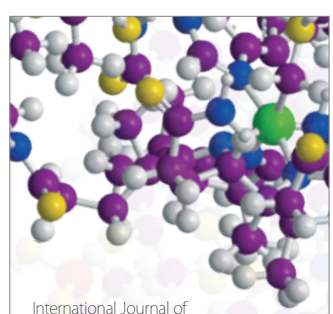

Carbohydrate Chemistry

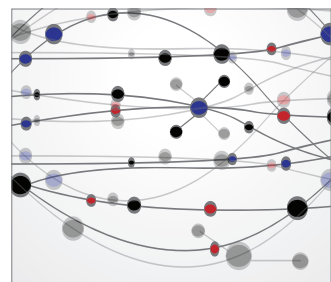

The Scientific World Journal
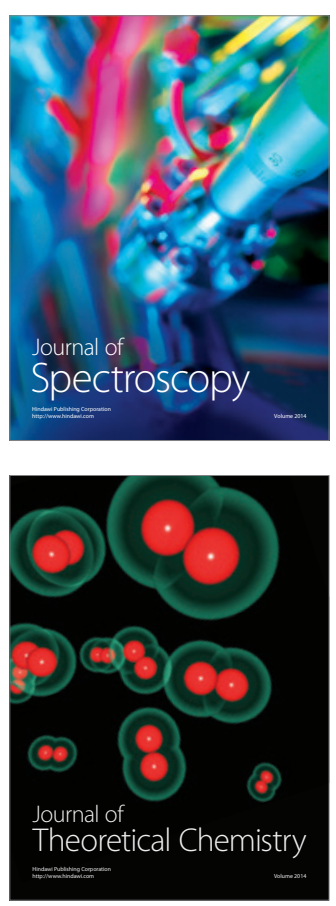
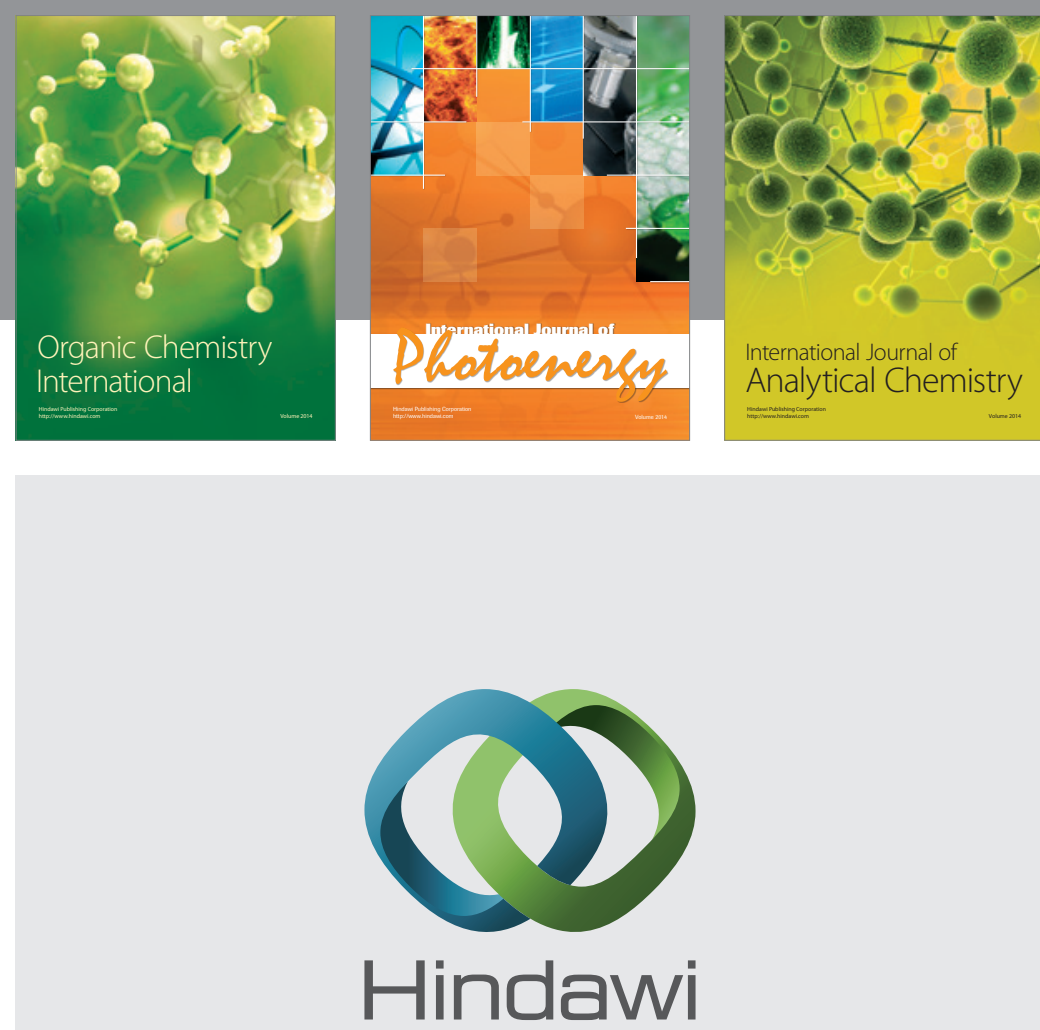

Submit your manuscripts at

http://www.hindawi.com
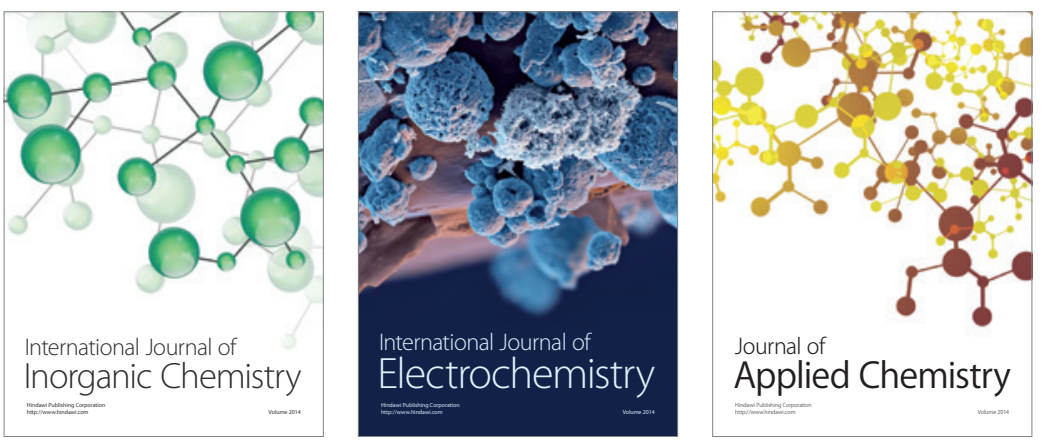

Journal of

Applied Chemistry
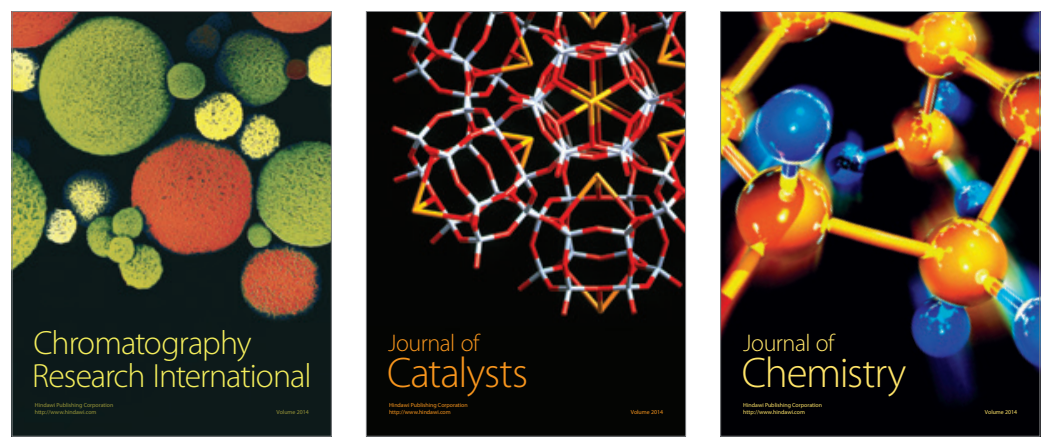
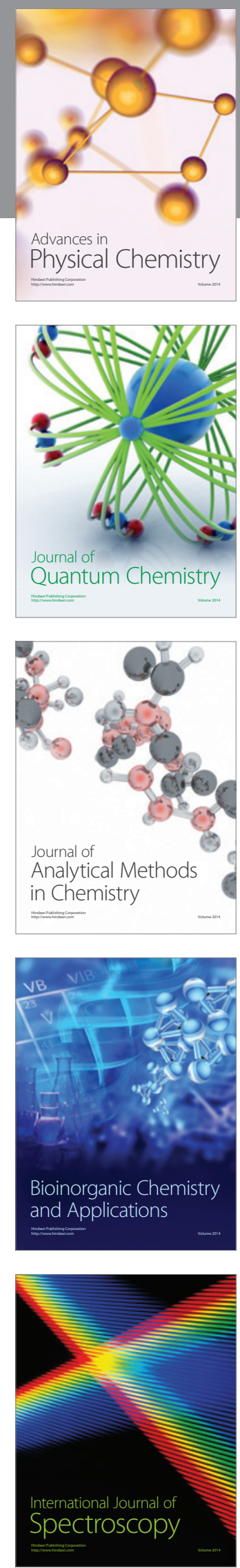